\title{
TB Control in Namibia 2002-2011: Progress and Technical Assistance
}

\author{
Jeroen van Gorkom ${ }^{*},{ }^{1}$, Farai Mavhunga ${ }^{2}$, Omer Ahmed Omer ${ }^{1}$, Amos Kutwa ${ }^{1}$, Abbas Zezai ${ }^{1}$, \\ Nunurai Ruswa ${ }^{1}$, Panganai Dhliwayo ${ }^{5}$, Souleymane Sawadogo ${ }^{3}$, Bert Schreuder ${ }^{1}$, \\ Rosalia Indongo 4 and Ella Shihepo ${ }^{2}$
}

\author{
${ }^{1}$ KNCV Tuberculosis Foundation, The Hague, The Netherlands \\ ${ }^{2}$ National Tuberculosis and Leprosy Program, Ministry of Health and Social Services, Windhoek, Namibia \\ ${ }^{3}$ United States Centers for Disease Control and Prevention (CDC), Windhoek, Namibia \\ ${ }^{4}$ United States Agency for International Development (USAID) Mission, Windhoek, Namibia \\ ${ }^{5}$ United States Centers for Disease Control and Prevention (CDC), Harare, Zimbabwe
}

\begin{abstract}
Namibia is among the five countries worstly affected by tuberculosis (TB) with a notification rate of 465/100,000 in 2011. This paper describes how the National Tuberculosis Leprosy Program (NTLP) developed from a poorly performing TB control program in 2002 into a well performing program in 2011.

The program achieved $85 \%$ treatment success for new sputum-positive patients, high coverage and performance on TB/Human Immunodeficiency Virus (HIV) collaborative program activities, and institution of Programmatic Management of Drug-Resistant Tuberculosis (PMDT) where this was absent before.

Provision of significant short- and long-term technical assistance provided by KNCV Tuberculosis Foundation (KNCV) in the period 2002-2011 was catalytic in leveraging a total of U\$ 80million of external funding in a stepwise approach balanced with absorption capacity, in combination with national policy review and support for its implementation on the basis of two consecutive costed national strategic plans.

The technical assistance by KNCV, in partnership with other international technical agencies for specialized areas, proved to work very well in this context where Namibian human resources and funding for TB control were initially limited and the Ministry of Health and Social Services (MoHSS) welcomed extensive technical assistance.
\end{abstract}

Keywords: Community-based tuberculosis control, HIV/AIDS, KNCV, MDR-TB, TB/HIV, technical assistance, tuberculosis.

\section{INTRODUCTION}

Namibia has one of the highest per capita prevalence rates for both tuberculosis (TB) and Human Immunodeficiency Virus (HIV) in the world. In 2011, it ranks as $4^{\text {th }}$ worstly affected country in the world in terms of TB notification rates per 100,000 population [1]. After adopting the Directly Observed Treatment Short-course (DOTS) strategy in 1995, the National Tuberculosis Leprosy Program (NTLP) in the Ministry of Health and Social Services (MoHSS) provided for short-course chemotherapy of new and retreatment patients, treatment for Multi-Drug Resistant TB (MDR-TB) (using amikacin, ethionamide, ciprofloxacin as second-line drugs), bacteriological examination services, and recording and reporting along World Health Organization (WHO) formats.

In 2002 the notification rate for TB (all forms) was $768 / 100,000$, with a reported treatment success rate of $68 \%$ for new sputum-smear positive TB patients and $64 \%$ for TB patients on re-treatment [2]. TB drug resistance prevalence

*Address correspondence to this author at the KNCV Tuberculosis Foundation, PO Box 146, 2501 CC The Hague, The Netherlands;

Tel: +31704270978; E-mail: vangorkomj@kncvtbc.nl was unknown in the absence of a national survey or laboratory drug resistance surveillance system. Patients were being treated for MDR-TB, but there were no data on notifications, treatment outcomes and drug resistance profiles. An HIV prevalence survey conducted by MoHSS in 1999 showed an HIV prevalence of $45 \%$ in TB patients and $19.3 \%$ in the adult population [3]. In 2002 only $12 \%$ of notified TB patients were reported with an HIV test result, of which 51\% were HIV-positive [2]. MoHSS provided Voluntary HIV Counseling and Testing services, as well as Cotrimoxazole Preventive Treatment (CPT) and treatment of other Opportunistic Infections through its integrated medical care system. Anti-Retroviral Therapy (ART) coverage of eligible People Living with HIV (PLHIV) was only $16 \%$ in 2004, and unknown but much lower than that for TB patients [4]. Two Non-Governmental Organizations (NGO's) were implementing community-based TB control in two regions with external financial support direct to a national NGO (in Windhoek by Penduka and Norwegian Lung Health Association; in Omaheke region by the Omaheke Health and Education Program and OXFAM-UK).

In general, human resources for health in 2002 were scarce, resulting in many vacant positions for nurses and one 
vacancy for a medical doctor in NTLP. NTLP at national level comprised one full time nurse, with 3 vacant positions. The National AIDS Control Program (NACOP) at national level equally lacked human resources and comprised 3 people in staff (one doctor, 2 nurses). In that year, NTLP, NACOP and the National Malaria Program were brought under one umbrella in the Directorate of Special Programs (DSP). Although housed in the same unit, coordination and collaboration between NACOP and NTLP were minimal. At regional level, HIV/AIDS, TB and Malaria control responsibilities are carried out by one or two nurses (2 positions in each region). At district level, within the primary health care services, nurses rotated every 3 months in the various departments of the health facility, with no dedicated staff for the TB clinic. There was no focused in-service training for TB case management and monitoring and evaluation for doctors and nurses. Quality of care to TB patients was inadequate, as shown by high defaulter and case fatality rates and a low treatment success rate; quality of care to MDR-TB patients was unreported/unknown.

By 2002 NTLP at national level was not receiving any external funding. Short-term technical assistance (TA) was being provided by WHO. NACOP was receiving support from the Presidents Emergency Program For AIDS Relief (PEPFAR) in the form of technical and financial assistance to the Namibia Institute of Pathology (NIP) for strengthening of clinical laboratory services for HIV/AIDS.

\section{TECHNICAL ASSISTANCE BY KNCV TUBER- CULOSIS FOUNDATION}

In 2002 MoHSS welcomed KNCV Tuberculosis Foundation (KNCV) to provide technical assistance to the NTLP through a KNCV consultant being resident in Namibia. MoHSS and KNCV developed a Memorandum of Understanding for this purpose to underline the long-term nature of this commitment and support to NTLP.

Between 2002-2005, KNCV provided short-term technical assistance focusing on resource mobilization, updating of national policy guidelines for DOTS, MDR-TB and TB/HIV, and development of a costed National Strategic Plan [5]. The United States Agency for International Development (USAID) and PEPFAR started providing TA through the Tuberculosis Coalition for Technical Assistance (TBCTA) mechanism (2004-2005). By May 2005, one full time resident KNCV consultant was based at the NTLP, in addition to ongoing short-term TA.

In the period 2005-2011 KNCV supported development of a second Medium-term Development Plan [6] and additional resource mobilization. In $2007 \mathrm{KNCV}$ registered in the country and established an office embedded next door to NTLP in MoHSS. KNCV technical staff increased to four resident medical officers and three support staff, while Short-Term Technical Assistance continued and expanded.

All TA activities by KNCV were carried out in close collaboration and support from other international organizations, including USAID and PEPFAR. Financial support was provided through international funding mechanisms: the Tuberculosis Control Assistance Program (TB CAP 2005-2010) and TB CARE I (2010 onwards).

\section{METHODS}

KNCV worked with NTLP using a long-term strategic approach, based on technical assistance for knowledge transfer, capacity building, and partnership building. Top priority was given to resource mobilization, strategic planning and budgeting, national policy update to latest international standards, monitoring/evaluation and supervision, and increase of human resources for TB control using both domestic and external funding. Long- and shortterm TA was provided for filling the gap in technical management and implementation capacity at the national level, as well as emphasizing strengthening of human capacity in the NTLP at all levels.

Main changes introduced in this period were: revision and strengthening of recording, monitoring and evaluation, implementation of the first national drug resistance survey, introduction of Fixed-Dosage Combination tablets for adults and children, developing the programmatic management of drug-resistant TB (PMDT), and development of a first national TB training module.

\section{RESULTS}

\section{Resource Mobilization}

During the period 2002-2011 KNCV leveraged U\$79.6m of donor funding in support of implementation of two NTLP National Strategic Plans 2004-2009 and 2010-2015 (Table 1).

\section{Human Resources}

The increase of NTLP financial resources resulted in an increase of staffing levels at national level from one public health nurse in 2002 to a staff compliment of eight in 2011, with four of these positions being donor funded (Global Fund and PEPFAR). Two health program administrators (nurses) were appointed in every region to coordinate TB, HIV and malaria activities, and two special disease program nurses were assigned to address TB, HIV and malaria related issues at district level as per MoHSS staff establishment, partially funded from GFATM.

Table 1. Resource Mobilization in Support of NTLP Namibia 2002-2011

\begin{tabular}{|c|c|c|c|c|c|c|c|c|c|c|c|}
\hline U\$ & $\mathbf{2 0 0 2}$ & $\mathbf{2 0 0 3}$ & $\mathbf{2 0 0 4}$ & $\mathbf{2 0 0 5}$ & $\mathbf{2 0 0 6}$ & $\mathbf{2 0 0 7}$ & $\mathbf{2 0 0 8}$ & $\mathbf{2 0 0 9}$ & $\mathbf{2 0 1 0}$ & $\mathbf{2 0 1 1}$ & Total \\
\hline \hline DGIS /NL* & $45 \mathrm{k}$ & $45 \mathrm{k}$ & $50 \mathrm{k}$ & $60 \mathrm{k}$ & - & - & - & - & - & - & $200 \mathrm{k}$ \\
\hline USAID/PEPFAR & - & - & $118 \mathrm{k}$ & $580 \mathrm{k}$ & $948 \mathrm{k}$ & $2.0 \mathrm{~m}$ & $2.3 \mathrm{~m}$ & $3.2 \mathrm{~m}$ & $2.6 \mathrm{~m}$ & $2.1 \mathrm{~m}$ & $13.8 \mathrm{~m}$ \\
\hline GFATM & $1.8 \mathrm{~m}$ & & - & $17.8 \mathrm{~m}$ & - & - & - & - & $37 \mathrm{~m}$ & - & $56.6 \mathrm{~m}$ \\
\hline \multicolumn{8}{|c|}{ Total } \\
\hline
\end{tabular}

\footnotetext{
* Directorate General International Collaboration, Netherlands.
} 
Table 2. Numbers of Health Workers Trained, NTLP Namibia 2004-2011

\begin{tabular}{|c|c|c|c|c|c|c|c|}
\hline & 2004 & 2005 & 2006 & 2007 & $2008-2010$ & 2011 & Total \\
\hline Number of doctors, pharmacists, nurses and Field Promoters (community-based TB workers) & 21 & 89 & 529 & 1,069 & 1,969 & 636 & 4,313 \\
\hline
\end{tabular}

Human resources for HIV/AIDS control and care delivery expanded with Global Fund and PEPFAR funding, requiring recruitment of considerable numbers of foreign doctors and nurses, resulting in a rapid scale-up of VCT, PMTCT and pre-ART care and ART.

\section{Training of Doctors and Nurses}

The funding enabled massive training of doctors and nurses and NTLP staff at all levels on the updated National TB Guidelines. This was done in close collaboration with ITECH. This high number was required to remedy the absence of any previous in-service training, the continuous high-turnover of staff in NTLP and in clinical care, the many foreign doctors in the health system involved in TB care, and the need for training of Field Promoters (community-based health care workers for TB). A total of 4,313 staff was trained in the period 2004-2011 (Table 2).

\section{Monitoring, Evaluation, Supervision and Surveillance}

Institution of quarterly district TB review meetings, sixmonthly regional TB review meetings, and more regular field supervision at all levels, resulted in much better timeliness and accuracy of $\mathrm{TB}$ recording and reporting, as well as data analysis and dissemination by the national level. This assisted NTLP staff in better annual planning based on analysis of reliable data. Data was reconstituted from field reports including an electronic TB register (CDC Botswana, Botusa version). In 2005, NTLP introduced revised records, registers and patient cards and reporting forms based on WHO recording and reporting guidelines. This included TB and HIV information, and later in 2008, introduced MDRTB records/registers which have led to capturing relevant and reliable TB data. Health facility data are being recorded continuously and reported quarterly by the district TB/HIV/AIDS/malaria coordinator, sent to the regional TB/HIV/AIDS/malaria coordinator, and onwards to the central unit. Central Unit NTLP aggregates district data into one national data base, and provides feedback to districts and regions during review meetings. Annual reports are developed and disseminated, and sent to WHO and other relevant international partners [7].

The number of new and relapse patients notified increased from 13,812 in 2002 to 14,920 in 2005, after which there has been a steady annual decline (Fig. 1). In 2011 WHO reported the Case Detection Rate (CDR) for Namibia as progressing from 53\% to 82\% [8]. In the 2012 report WHO adjusted the CDR downwards to $40 \%$ in 2000 climbing to $64 \%$ in 2011 [1].

Treatment success of new sputum-smear positive TB patients increased from $56 \%$ in 2000 to $85 \%$ in the 2010 cohort (Fig. 2). The improvement was largely attributed to a decrease in the proportion of patients reported as 'Defaulted' from $15 \%$ to $4 \%$, as well as the proportion of patients reported as 'Not Evaluated' from $21 \%$ to $2 \%$. During the same period the treatment failure rate for new smear positive patients increased from 2 to $4 \%$.

Improvements in the treatment success rates for retreatment patients only started to be realized between 2005 and 2009. In this patient category, the treatment success rate increased from $54 \%$ in 2004 to $73 \%$ in 2009 . The improvement was largely due to a decrease in the proportion of patients who defaulted treatment from $13 \%$ to $6 \%$ as well as patients 'Not Evaluated' from 17\% to 3\%. The reported treatment failure rate increased from $6 \%$ to $9 \%$ during this period. The improvement of treatment success is a result of both improved program reporting (less misclassification) in combination with better patient support and follow-up, due to the increased coverage of CBTBC.

\section{Community-Based TB Control (CBTBC)}

CBTBC services function as an extension of MoHSS into the community, as MoHSS is not able to provide the necessary human resources for providing adequate patient

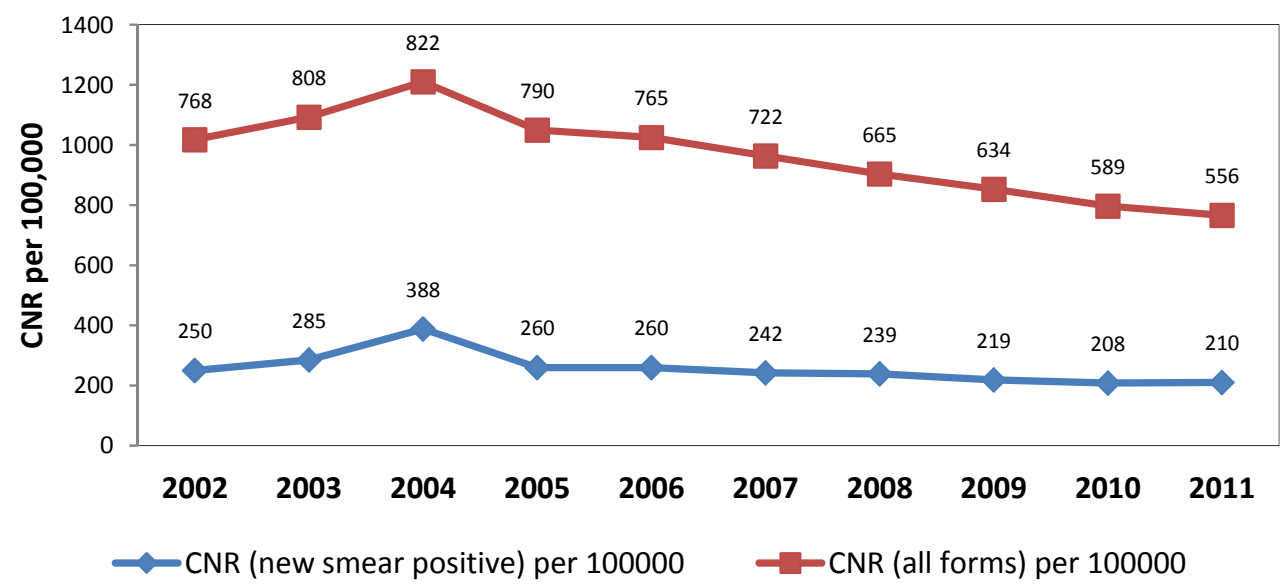

Fig. (1). Tuberculosis case notification rates (CNR), NTLP Namibia, 2002-2011. 


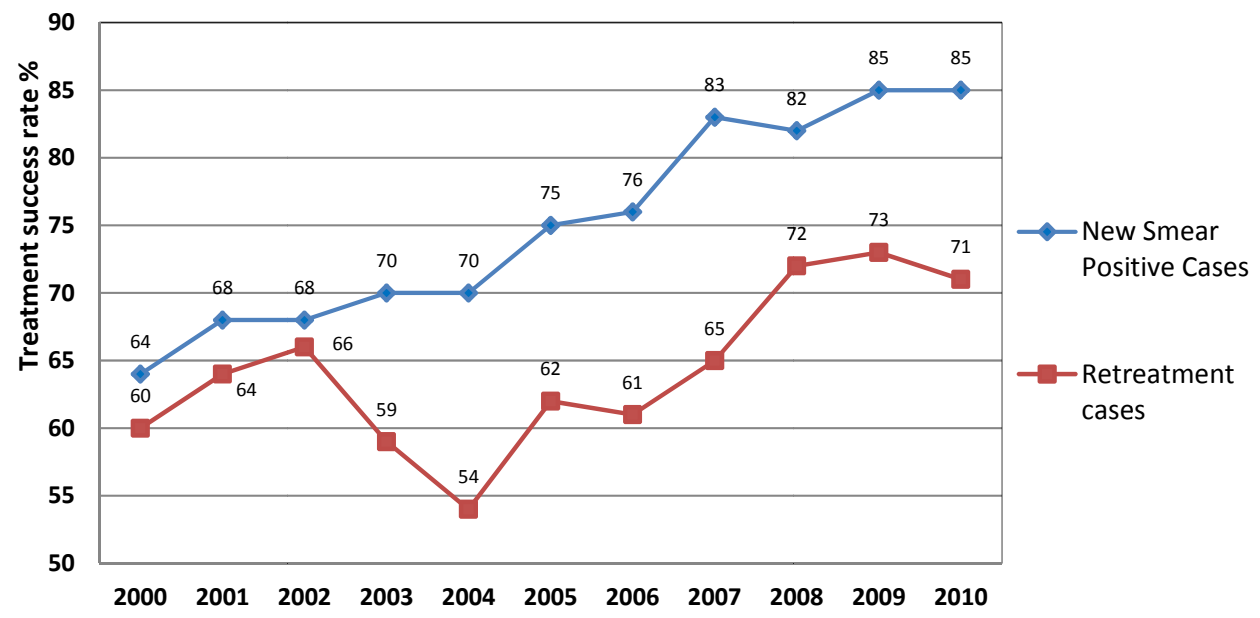

Fig. (2). Treatment success rates for new smear-positive and retreatment TB patients, NTLP, Namibia, 2000-2010.

support in and beyond the health facility, by lack of a cadre of 'community field worker' that operates from a health facility and reaches out into the homes of patients. The increased financial resources from USAID/PEPFAR and GFATM enabled NTLP to expand CBTBC to 31 out of 34 existing districts in 2011 (Fig. 3). This was done mostly by contracting various NGO's in Namibia (CoHeNa, Health Unlimited, Johanitter Hilsfwerk, Penduka, Namibia Red Cross Society, Project HOPE and CESTAS). KNCV made a contract with two MoHSS district health authorities for the implementation of CBTBC. Each NGO used region- and district-specific approaches in achieving patient access through CBTBC, always working in close collaboration with and from existing MoHSS health facilities. All contracted Field Promoters (community-based volunteers) were tasked with patient education, Directly Observed Treatment (DOT), home visits, tracing of patients, as well as mobilization and involvement of treatment supporters in the patient's home.

\section{TB/HIV}

Expansion of TB/HIV collaborative activities was the result of joint strategic planning by the NTLP and NACOP, with funding largely provided by the GFATM (round 5 and 10) and PEPFAR.

In 2002 coverage of HIV testing among TB patients was reported low $(12 \%)$ as it was based on a Voluntary
Counseling and Testing approach (VCT). With increased funding and commitment by NTLP and NACOP, and by using the Provider Initiated Testing and Counseling (PITC) approach, testing levels increased fast to $84 \%$ of TB patients reported with an HIV result in 2011, of whom 50\% are HIV positive, $98 \%$ are on CPT and $54 \%$ are on ART (Fig. 4). In 2009 90,000 People Living with HIV (PLHIV) were screened for TB $(50 \%$ of those in care, $\mathrm{n}=180,000)$, and 13,989 PLHIV in care were reported as commenced on Isoniazid Preventive Therapy (IPT) - 8\% of total PLHIV registered in care [9].

In 2002 overall the HIV seroprevalence was $22 \%$ among pregnant women (age between 15-44 years). This decreased to $19.7 \%$ in $2004,19.9 \%$ in $2006,17.8 \%$ in 2008 , and $18.2 \%$ in 2012 [10]. A decreasing trend is seen in younger agegroups (15-29 of age) while the prevalence among older agegroups is showing a slight increase. Between 2002 and 2011 overall ART coverage rapidly expanded from $12 \%$ to $84 \%$ of those in need, with $41 \%$ of HIV positive women in the survey being on ART. By $201154 \%$ of public health facilities were providing ART to 102,000 individuals representing more than $80 \%$ of those in need (CD4<350ul) [11]. The steady decline of annual TB notifications since 2004 is likely to be the result of the declining HIV prevalence in the age-group 15-29, increasing ART coverage for eligible PLHIV, and the effect of TB control measures.

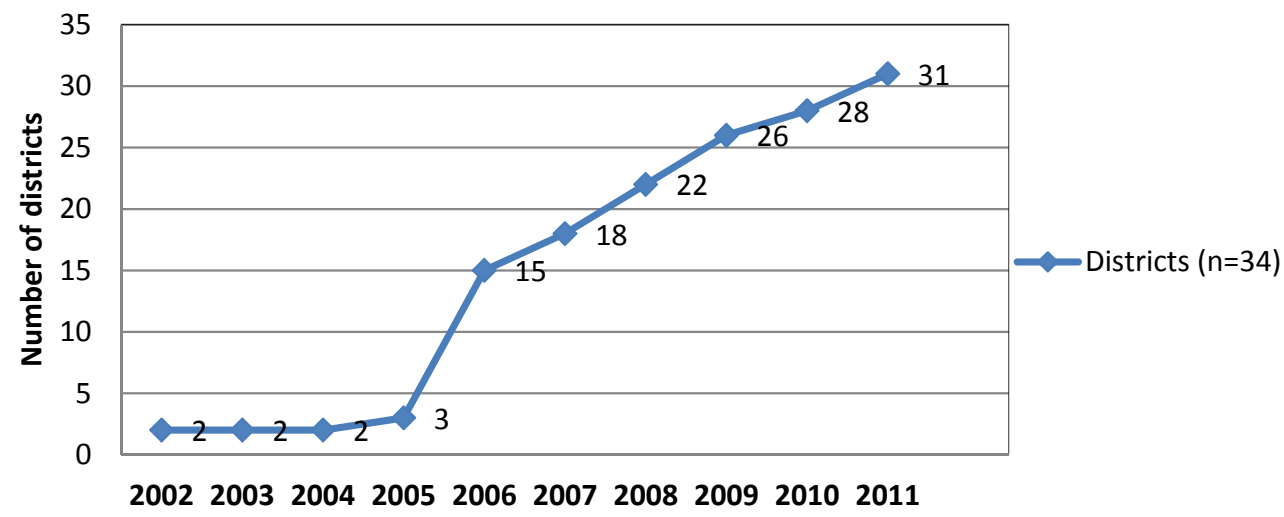

Fig. (3). Community-based TB Control coverage by district, NTLP, Namibia, 2002-2011. 


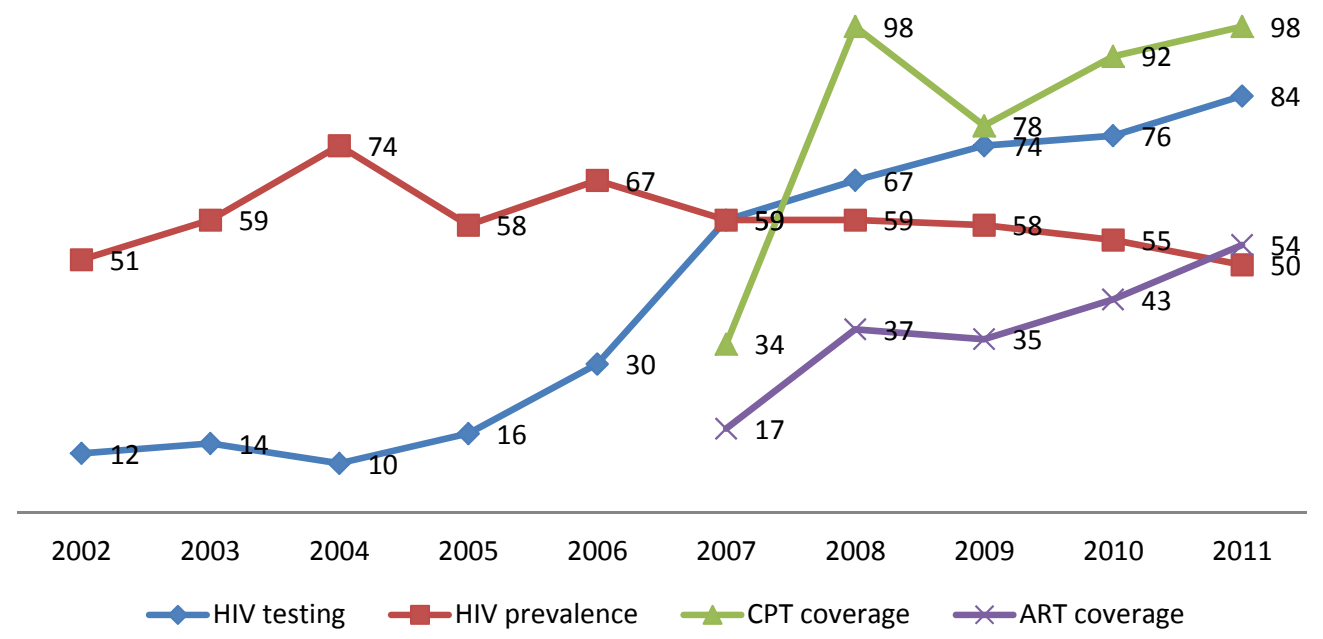

Fig. (4). TB/HIV performance indicators (\%), NTLP Namibia, 2002-2011.

Extensively- and Multi-Drug Resistant Tuberculosis (X/MDR-TB)

Using the WHO guidelines on PMDT, KNCV supported comprehensive supervision resulting in improved DR-TB case management at DR-TB centers, better notification and evaluation. Ten MDR-TB admission and referral facilities were established from only one before 2007. All patients are closely monitored and evaluated by a National DR-TB Expert Committee, which performs clinical audits of each patient, and participates in supervision of DR-TB sites. With support from TB CAP, KNCV funded and organized an MDR-TB training course in close collaboration with The Union since 2007. This course developed into a regional course attracting participants from all over Africa.

Data on notification of patients registered for treatment with drug-resistant $\mathrm{TB}$ and interim and final treatment outcomes did not exist before 2007. In the absence of a routine laboratory surveillance of drug-resistant $\mathrm{TB}$, the number of patients with a laboratory diagnosis of drugresistant TB - not registered for treatment - is unknown. Based on the prevalence of MDR-TB, it is expected that there are about 670 patients with MDR-TB emerging among notified new and retreatment patients with pulmonary tuberculosis each year [1], which implies that only about $36 \%(n=240 / 670)$ of incident MDR-TB patients were actually treated in 2011. Notification data for XDR- MDRand Policy Drug Resistant TB since 2007 show a steady decline after reaching a peak in 2009 (Fig. 5).

Final treatment outcomes of those patients registered with confirmed MDR-TB are shown in the Table 3. Maintaining completeness of data is a challenge as seen by the $\%$ of patients evaluated. A concern is the high proportion of patients whose treatment is continued after 24 months of treatment.

The first 6-months interim outcome results show an improvement in culture conversion rate from $61 \%$ in 2010 to $66 \%$ in 2011, as well as decline in death rate (Table 4).

\section{TB Drug Resistance Survey}

The first national TB drug resistance survey was conducted in 2008-2009, as a joint effort of NTLP/NIP/KNCV/CDC. Results showed that the MDR-TB

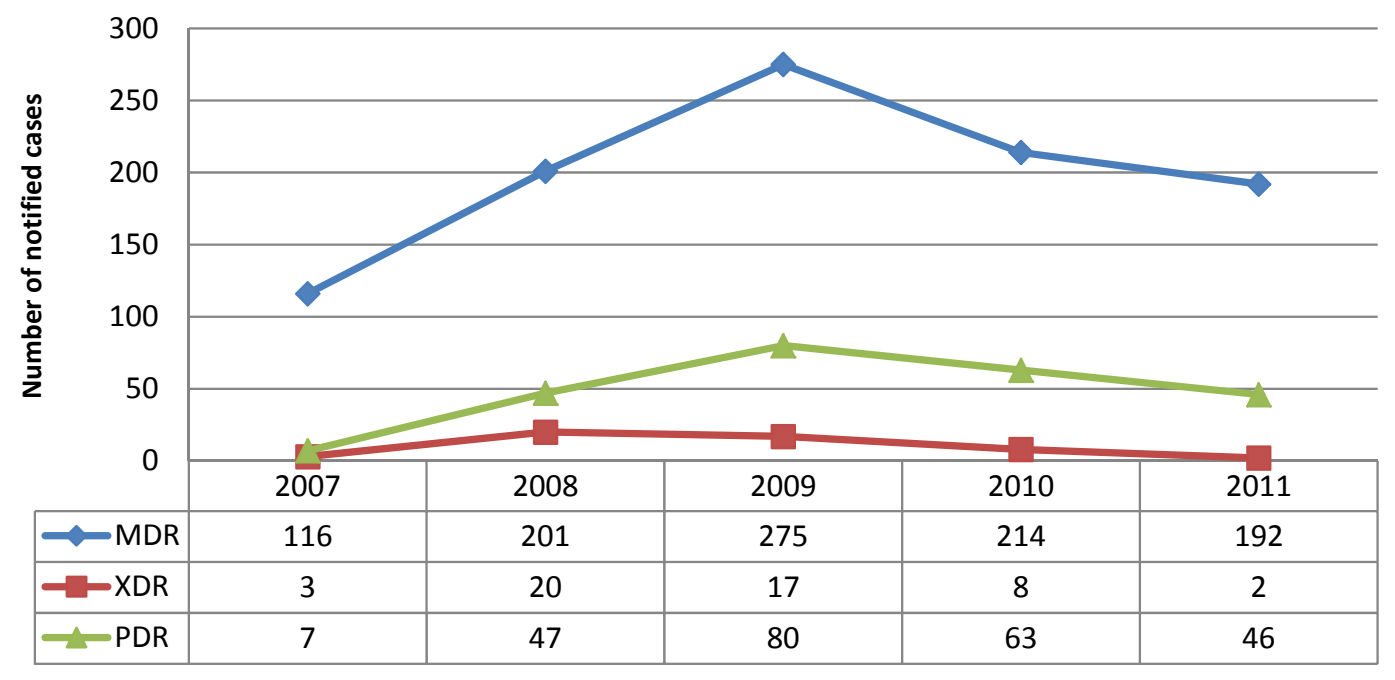

Fig. (5). Notifications of patients with drug-resistant TB registered for treatment, NTLP Namibia, 2007-2011. 
Table 3. MDR-TB treatment final outcome data NTLP Namibia, 2007-2009

\begin{tabular}{|c|c|c|c|c|c|c|c|c|c|c|c|c|c|c|c|c|c|}
\hline \multirow{2}{*}{ Cohort Period } & \multirow{2}{*}{ Notified } & \multicolumn{2}{|c|}{ Cured } & \multicolumn{2}{|c|}{ Completed } & \multicolumn{2}{|c|}{ Failed } & \multicolumn{2}{|c|}{ Died } & \multicolumn{2}{|c|}{ Default } & \multicolumn{2}{|c|}{ Transfer } & \multicolumn{2}{|c|}{ Rx Continued } & \multicolumn{2}{|c|}{ Evaluated } \\
\hline & & No & $\%$ & No & $\%$ & No & $\%$ & No & $\%$ & No & $\%$ & No & $\%$ & No & $\%$ & No & $\%$ \\
\hline 2007 & 116 & 32 & 28 & - & 0 & 11 & 9 & 11 & 9 & 26 & 21 & 38 & 33 & - & 0 & 116 & 100 \\
\hline 2008 & 201 & 46 & 21 & 43 & 20 & 22 & 10 & 49 & 22 & 23 & 11 & 35 & 16 & 0 & 0 & 218 & 108 \\
\hline 2009 & 275 & 78 & 32 & 37 & 15 & 4 & 1 & 51 & 21 & 40 & 16 & 9 & 4 & 27 & 11 & 246 & 89 \\
\hline
\end{tabular}

MDR-TB treatment success improved since $2007-2009$ from $28 \%$, to $41 \%$ and $47 \%$, which is still far short of the target of $75 \%$. Encouraging is the low failure and transfer rate in the 2009 cohort, but case-fatality and default rate remains a serious concern, as well as completeness of data.

Table 4. MDR TB First Six-Month Interim Outcomes, NTLP Namibia, 2010-2011

\begin{tabular}{|c|c|c|c|c|c|c|c|c|c|c|c|c|c|c|c|}
\hline \multirow{2}{*}{$\begin{array}{l}\text { Cohort } \\
\text { Period }\end{array}$} & \multirow{2}{*}{ Notified } & \multicolumn{2}{|c|}{ Culture Neg } & \multicolumn{2}{|c|}{ Culture Pos } & \multicolumn{2}{|c|}{ Failed } & \multicolumn{2}{|c|}{ Died } & \multicolumn{2}{|c|}{ Default } & \multicolumn{2}{|c|}{ Transfer } & \multicolumn{2}{|c|}{ Evaluated } \\
\hline & & No & $\%$ & No & $\%$ & No & $\%$ & No & $\%$ & no & $\%$ & No & $\%$ & No & $\%$ \\
\hline 2010 & 214 & 140 & 61 & 20 & 9 & 2 & 1 & 34 & 15 & 21 & 9 & 12 & 5 & 229 & 107 \\
\hline 2011 (9 months) & 192 & 105 & 66 & 13 & 8 & 1 & 1 & 16 & 10 & 14 & 9 & 14 & 9 & 158 (Jan-Sep) & 82 \\
\hline
\end{tabular}

prevalence among new patients was $3.8 \%$ and among previously treated patients $16.5 \%$ [12].

\section{Laboratory Strengthening}

Through the technical and financial support by CDC to NIP, NTLP could rely on more rapid and quality assured liquid culture instead of solid media. As a result the culture turnaround time improved from 6-8 months in 2006/2007 to 3-7 weeks in 2012.

\section{DISCUSSION}

NTLP performance indicators for DOTS, TB/HIV and MDR-TB have improved remarkably in the ten years period of KNCV support. This is caused by a successful combination of government commitment and sustained longand short term technical assistance provided by KNCV, directed at program strengthening and close collaboration with NTLP at all levels. After an initial period that KNCV funded its work from a Dutch Government grant, the Technical Assistance programs supported by USAID (TBCTA, TB CAP and TB CARE I) provided for a mechanism of coordinated technical assistance, involving various technical partners complementing each others' capacities (KNCV, MSH, The Union, WHO). The gradual increase of external financial resources to a total of U\$80 million leveraged by KNCV provided external funding for the implementation of two consecutive National Strategic Plans. This case demonstrates the successful interaction of technical assistance, resource mobilization and long-term strategic planning for program implementation, within a continuous cycle of policy review $=>$ strategy review $=>$ resource mobilization $=>$ implementation $=>M \& E$ and review $\Rightarrow$ policy revision etc. with technical assistance being a critical input at every step of the cycle. This could only be achieved thanks to MoHSS demonstrating leadership by welcoming and recognizing the need for technical assistance in all these aspects, providing for a continuous provision of clinical laboratory services and anti-tuberculosis medicines, filling all vacant government positions relevant to TB control at national, regional and district level, if necessary with foreigners, being responsive to newly emerging threats (XDR-TB), and by committing to the implementation of two consecutive Medium-Term Development Plans.

\section{CONCLUSIONS AND RECOMMENDATIONS}

This progress is impressive, yet many challenges remain for the future. According to WHO, case detection rate is still too low at $64 \%$ [1] and this can only be raised by the program giving more priority to case-detection, where it prioritized case-holding in the past decade. Current programmatic successes need to be sustained, their quality improved, and their coverage still expanded.

Access to TB/HIV care still leaves to be desired, with only $54 \%$ of HIV-positive TB patients being registered for ART, and only $58 \%$ of public clinics providing ART. The target of $100 \%$ ART for TB can only be achieved with further decentralization of ART delivery, to all TB treatment points. Although $82 \%$ of TB patients are registered with an HIV test result, this is still below the target of $100 \%$. Intensified Case Finding and provision of Isoniazid Preventive Treatment to PLHIV is being increasingly reported, but is still low.

Access to diagnosis and treatment for drug resistant TB is still a challenge. Based on the drug resistance survey, only $36 \%$ of annually emerging patients with MDR-TB were diagnosed and registered for treatment in 2011. An important reason for this is that a considerable number of Category II patients (patients having been treated before with $16.5 \%$ prevalence of MDR-TB) are not examined with culture/drug sensitivity testing at the start of treatment as NTLP Guidelines prescribe (source NTLP, data unavailable). The highly centralized nature of culture and drug sensitivity testing services in the capital Windhoek presents a logistical challenge in this matter. Countrywide access to rapid diagnostic tests (e.g. GeneXpert MTB/Rif) may enable NTLP to diagnose rifampicin resistance much quicker in a decentralized manner, and start patients on an appropriate empiric second-line treatment regimen much earlier, while 
waiting for a full drug resistance profile and adjustment of the regimen.

NLTP is highly dependent on external funding for human resources at national level and for implementing CBTBC; progress can rapidly be lost when external funding starts diminishing without other (domestic) funding for TB control increasing adequately. NACOP is highly dependent on external funding for hiring foreign nurses and doctors to provide HIV/AIDS care. A too radical decline of external funding may result in decrease of treatment success and case detection, TB/HIV scale-up risks to slow down or stop altogether, access to diagnosis and treatment of MDR-TB will remain limited, and result in poor treatment outcomes contributing to further development of extensively drugresistant tuberculosis.

Reduction of donor funds may also negatively affect technical assistance provided by KNCV and other partners. MoHSS and technical partners need to intensify their efforts of capacity transfer to preferably Namibian (nationality) counterparts before funding for technical assistance is reduced, and support NTLP in advocacy to MoHSS for good evidence-based financial domestic commitments and investments for TB control.

\section{CONFLICT OF INTEREST}

The authors confirm that this article content has no conflict of interest.

\section{ACKNOWLEDGEMENTS}

MoHSS, NTLP, NACOP staff; USAID and PEPFAR through TBCTA, TB CAP, TB CARE I; National partners in Namibia for TB control; TBCTA partners (MSH, The Union, WHO); CDC.

\section{REFERENCES}

[1] Global Tuberculosis Report 2012. Switzerland: WHO 2012.

[2] NTLP Annual Report 2002, Namibia: MoHSS 1999.

[3] National HIV Surveillance Report 1999. Namibia: MoHSS 1999.

[4] National AIDS Control Program Annual Report 2002. Namibia: MoHSS 2002.

[5] Tuberculosis Medium Term Development Plan, MTP I, 2004-2009. Namibia: MoHSS 2009.

[6] Tuberculosis Medium Term Development Plan, MTP II, 20102014. Namibia: MoHSS 2013.

[7] NTLP Annual Reports 2002-2011. Namibia: MoHSS 2011.

[8] Global Tuberculosis Report 2011. Switzerland: WHO 2011.

[9] Available at: http://www.unaids.org/en/regionscountries/countries /namibia/ [Cited: $13^{\text {th }}$ Aug 2012].

[10] National HIV Surveillance report. Namibia: MoHSS/NACOP 2012

[11] ART Quarterly Report 2012. Namibia: MOHSS/NACOP 2012.

[12] National Tuberculosis Drug Resistance Survey 2010. Namibia: MoHSS. 